\title{
Prescrições medicamentosas de pacientes atendidos no ambulatório de geriatria de um hospital universitário: estudo transversal descritivo
}

\author{
Drug prescriptions of patients treated in a geriatric outpatient ward \\ of a university hospital: a descriptive cross-sectional study
}

\author{
Vanessa da Silva Cuentro ${ }^{1}$ \\ Marcieni Ataide de Andrade ${ }^{1}$ \\ Letícia Farias Gerlack ${ }^{2}$ \\ Ângelo José Gonçalves Bós ${ }^{3}$ \\ Marcos Valério Santos da Silva ${ }^{1}$ \\ Alex Ferreira de Oliveira ${ }^{4}$
}

\begin{abstract}
The scope of this study was to evaluate drug prescriptions for elderly patients in the geriatric outpatient ward of a hospital. A cross-sectional descriptive study was conducted in a university hospital, with the application of a questionnaire. Patients aged $\geq 60$ years were interviewed about their drug prescriptions and a total of 208 patients were interviewed. The average number of drugs used per person was 3.8 and the drugs most used were for the cardiovascular system (37\%). 406 potential interactions were identified in 140 patients (67.3\%), and duplicate therapy was found in two prescriptions. The potentially inappropriate drug most used for the elderly was nifedipine (2.4\%). Data from this study support the relevance of the issue of drug use among the elderly, thus reinforcing the importance of the evaluation of the process of the prescription of drugs for this population.
\end{abstract}

Key words Elderly individuals, Drug prescriptions, Drug evaluation
Resumo O objetivo foi avaliar prescrições medicamentosas de pacientes idosos atendidos no ambulatório de geriatria de um hospital. Método: Foi realizado um estudo transversal e descritivo, desenvolvido em um hospital universitário, com o auxílio de um questionário. Os pacientes com idade $\geq 60$ anos foram entrevistados sobre os medicamentos prescritos. Resultados: Foram entrevistados 208 pacientes. A média de medicamentos utilizados por pessoa foi de 3,8 e os medicamentos mais utilizados foram do aparelho cardiovascular (37,0\%). Foram identificadas 406 interações potenciais em 140 pacientes (67,3\%) e duplicidade terapêutica em duas prescrições. O medicamento potencialmente inapropriado para idosos mais utilizado foi o nifedipino (2,4\%). Conclusão: Os dados deste estudo corroboram sobre a temática do uso de medicamentos em idosos, reforçando a importância da avaliação do processo de prescrição de medicamentos para essa população.

Palavras-chave Idoso, Prescrições de medicamentos, Avaliação de medicamentos
${ }^{1}$ Faculdade de Farmácia, Universidade Federal do Pará. R. Augusto Corrêa 1, Guamá. 66.075-110 Belém PA Brasil.vancuentro@ hotmail.com

${ }^{2}$ Universidade de Brasília.

${ }^{3}$ Instituto de Geriatria e

Gerontologia, PUC/RS.

${ }^{4}$ Hospital Universitário João de Barros Barreto. 


\section{Introdução}

Avanços da medicina e melhorias nas condições gerais de vida da população levaram à redução da taxa de natalidade e ao aumento da expectativa de vida, contribuindo para o envelhecimento populacional. O número de habitantes com sessenta anos ou mais passou de 3 milhões em 1960 para cerca de 21 milhões em 2009, devendo atingir 32 milhões em $2020^{1}$. Esse aumento da população idosa colabora para a maior prevalência de inúmeras e variadas patologias, cujos tratamentos em geral incluem recursos farmacológicos ${ }^{2}$, que levam a prática de polifarmácia, definida como uso de vários medicamentos simultaneamente ${ }^{3,4}$, fator esse que tem grande impacto na segurança do paciente idoso, tendo em vista que a polifarmácia é a grande responsável pelas reações adversas a medicamentos (RAM) e interações medicamentosas ${ }^{2,5,6}$.

Uma interação medicamentosa (IM) ocorre quando os efeitos e/ou a toxicidade de um fármaco são alterados pela presença de outro, podendo ser benéfica (aumento da eficácia) ou maléfica (diminuição da eficácia e/ou aumento da toxicidade) ${ }^{7}$. As interações podem ser do tipo: farmacocinéticas (alterações na absorção, distribuição, metabolismo e excreção) ou farmacodinâmicas (sinergismo ou antagonismo da ação) ${ }^{8}$.

Além da polifarmácia e interação medicamentosa, há características clínicas importantes da população idosa. $O$ fato de estes indivíduos apresentarem uma série de alterações que interferem diretamente nos processos farmacocinéticos dos fármacos ${ }^{9}$. Nesse contexto, algumas categorias de medicamentos passaram a ser impróprias para o idoso, seja por falta de eficácia terapêutica ou por risco aumentado de efeitos adversos ${ }^{10}$.

Os medicamentos são considerados potencialmente inapropriados em idosos quando os riscos de seu uso superam seus benefícios ${ }^{11,12}$. Atualmente, as prescrições inapropriadas para este segmento constituem práticas irracionais ao consumo de medicamentos ${ }^{12,13}$. Há na literatura uma ferramenta bem descrita e validada para avaliação da prescrição de medicamentos em idosos, determinada em consenso: o critério de Beers ${ }^{14-17}$. Em 1991, Mark Beers desenvolveu junto com especialistas o primeiro conjunto de critérios para identificar o uso inadequado de medicamentos por idosos institucionalizados nos Estados Unidos. Estes profissionais definiram, de maneira consensual, uma lista de 19 medicamentos inadequados e 11 medicamentos cuja dose, frequência de uso ou duração do tratamento eram inadequadas ${ }^{14}$. A lista inclui sedativos e hipnóticos, analgésicos, antipsicóticos, antidepressivos, anti-hipertensivos, anti-inflamatórios não esteroides e hipoglicemiantes orais. Em 1997, esta lista foi atualizada, incluindo novos fármacos e incorporando novas evidências da terapia farmacológica ${ }^{15}$. A terceira revisão dos critérios de Beers ocorreu no ano de 2003, na qual foram identificados, por consenso, 48 medicamentos ou classes de medicamentos inadequados e uma lista de medicamentos inadequados em indivíduos com 20 condições patológicas específicas ${ }^{16}$. Na versão final dos Critérios de Beers que ocorreu neste ano de $2012^{17}$ foi utilizada uma abordagem baseada em evidências, incluindo 53 medicamentos, ou classes de medicamentos, divididos em três categorias: medicamentos potencialmente inadequados e classes a evitar em idosos, medicamentos potencialmente inadequados e classes a evitar em idosos com certas doenças e síndromes que podem ser exacerbadas pelos medicamentos listados e, por fim, medicamentos a serem usados com cautela em idosos.

Diante destes fatores, polifarmácia, interação medicamentosa e medicamento inapropriado, é fácil perceber que a prescrição de medicamentos no idoso é extremamente complexa ${ }^{18}$. Portanto, o uso de medicamentos por idosos é um tema bastante abordado em estudos realizados no Brasil $^{2,19-23}$, suas consequências podem ser consideradas um importante problema de saúde pública, pois estão relacionadas ao aumento da morbimortalidade ${ }^{6}$.

Logo, considerando a importância que representa o uso correto de medicamentos entre a população idosa, propomos neste trabalho caracterizar o perfil sociodemográfico dos idosos, verificar as classes de medicamentos mais utilizadas e analisar as prescrições medicamentosas de pacientes idosos atendidos no ambulatório de geriatria de um hospital universitário.

\section{Métodos}

\section{Tipo de estudo}

Foi realizado um estudo transversal, observacional e descritivo, desenvolvido no Hospital Universitário João de Barros Barreto (HUJBB), da Universidade Federal do Pará, no município de Belém, Pará - Brasil, entre dezembro de 2010 a maio de 2011.

\section{Local da pesquisa}

O HUJBB é um hospital escola, de nível terciário, com capacidade para 300 leitos e integrante 
do Sistema Único de Saúde (SUS). Os pacientes atendidos no HUJBB, em sua maioria, são oriundos das Unidades Básicas de Saúde (UBS), nível primário de atendimento, e encaminhados aos diferentes ambulatórios do hospital, como Clínica Médica, Pneumologia, Infectologia, Endocrinologia, Cardiologia e Geriatria. O local de estudo do presente trabalho é o Setor de Geriatria.

\section{Processo de Amostragem e amostra}

O processo de amostragem foi de natureza sistemática. Para determinação do tamanho da amostra, foi considerado um erro amostral de 5\% e nível de confiança de 95\%, tomando como base o número de pacientes idosos atendidos em 2009 que totalizou 450 pacientes, com base nesses parâmetros, foi definida uma amostra de 208 indivíduos. Foi utilizado o método de amostragem aleatória simples sem reposição para seleção da amostra deste estudo, no programa Biostat 5.0.

\section{Critério de Inclusão}

O critério de inclusão utilizado foram idosos com idade igual ou superior a 60 anos que possuíssem no momento da entrevista prescrição médica.

\section{Critério de Exclusão}

Pacientes com idade inferior a 60 anos.

\section{Variáveis do Estudo}

\section{Dependente}

A variável dependente do estudo foi o número de medicamentos prescritos. Após a identificação, os medicamentos foram desdobrados em seus princípios ativos e classificados de acordo com Anatomical Therapeutical Chemical Code (ATC) da $\mathrm{OMS}^{24}$, em categorias terapêuticas, conforme o primeiro (anatômico) e segundo níveis da classificação (terapêutico).

\section{Independentes}

As variáveis independentes incluíram: sexo, idade e procedência.

\section{Coleta de dados}

Os pacientes foram selecionados na sala de acolhimento da consulta médica sendo orientados sobre a pesquisa, tendo a liberdade de escolha em participar ou não da mesma. Para os que aceitaram em participar, foi solicitado o preenchimento do
Termo de Consentimento Livre e Esclarecido, elaborado em duas vias, ficando uma para o entrevistado e outra para a entrevistadora.

As informações foram obtidas através de um formulário semiestruturado elaborado pelos pesquisadores contendo dados como nome, idade, gênero, procedência e medicamentos prescritos. Para a coleta de informações sobre os medicamentos foi solicitado ao idoso que mostrasse todas as suas prescrições no momento da entrevista.

Quando o idoso não estava apto a responder perguntas (por exemplo: o paciente precisava de ajuda para fornecer as informações ou quando não foi possível coletar qualquer informação diretamente com o entrevistado), estas eram conferidas e/ou colhidas de seus cuidadores.

\section{Análise dos dados}

A análise das prescrições medicamentosas de pacientes idosos baseou-se nos seguintes parâmetros:

- Existência de polifarmácia: considerou-se a polifarmácia como o uso de cinco ou mais medicamentos simultaneamente ${ }^{25}$;

- Interações medicamentosas: a partir do perfil de medicamentos prescritos, as interações droga-droga foram identificadas e classificadas quanto à gravidade, conforme DrugReax ${ }^{\circledR}$ System do Micromedex ${ }^{\circledR 26} \mathrm{em}$ : maior - a interação pode ter risco de morte e/ou requer intervenção médica; moderada - a interação pode resultar em uma exacerbação da condição do paciente e/ ou requer uma alteração na terapia; e menor - a interação pode ter efeitos clínicos limitados, e as manifestações podem incluir um aumento na frequência ou gravidade dos efeitos colaterais, mas geralmente pode-se não requerer maior alteração na terapia;

- Prescrições de medicamentos considerados inapropriados segundo critério de Beers: o uso de medicamentos inadequados foi classificado segundo versão atualizada do critério de Beers ${ }^{17}$;

- Existência de duplicidade terapêutica: consideradas como o uso simultâneo de dois fármacos do mesmo subgrupo terapêutico, de acordo com a classificação Anatômica-Terapêutica-Clínica (ATC), proposta pela Organização Mundial da Saúde ${ }^{24}$.

A organização e o registro dos dados foram realizados no programa Excel ${ }^{\circledR} 2010$. Para análise estatística, foram utilizados os softwares EpiInfo 3.5.3. O método de análise inclui a estatística descritiva (média, desvio padrão, frequências relativas e absolutas), considerando-se um intervalo de confiança de $95 \%$. 


\section{Procedimentos éticos}

O trabalho teve início após a aprovação do Comitê de Ética e Pesquisa do Hospital Universitário, respeitando-se os preceitos de pesquisa envolvendo seres humanos, de acordo com a resolução CNS n $196 / 96^{27}$.

\section{Resultados}

Todos os idosos abordados optaram por participar da pesquisa, não havendo desistências, totalizando 208 entrevistados.

\section{Características sociodemográficas}

Foram analisadas prescrições de 208 pacientes da instituição, a média de idade desses pacientes foi de 73,4 anos $\pm 8,8$ sendo $66,8 \%$ mulheres e $33,6 \%$ homens, e a maior parte desses pacientes $(96,2 \%)$ é oriunda da capital (Tabela 1).

\section{Caracterização do uso de medicamentos}

Os medicamentos mais utilizados, reunidos por grupamento anatômico segundo classificação ATC, foram do sistema cardiovascular $(37,4 \%)$, sistema nervoso central $(27,5 \%)$ e sistema digestório e metabólico $(14,1 \%)$, estes representaram juntos $79,0 \%$ dos medicamentos prescritos. No sistema cardiovascular, os fármacos mais usados são pertencentes ao subgrupo que atua sobre o sistema renina-angiotensina (14,8\%), diuréticos $(7,3 \%)$ e bloqueadores seletivos de canais de cálcio $(4,7 \%)$. No grupamento referente ao sistema

Tabela 1. Distribuição das características sociodemográficas dos idosos atendidos $(\mathrm{n}=208)$ no AG/HUJBB, 2011.

\begin{tabular}{lrr}
\hline $\begin{array}{c}\text { Características } \\
\text { Sociodemográficas }\end{array}$ & $\mathbf{n}(\%)$ & $\left(\mathbf{I C}^{*} \mathbf{9 5} \%\right)$ \\
\hline Faixa etária & & \\
$60-69$ anos & $80(38,5)$ & $(31.8-45.4)$ \\
$70-79$ anos & $72(34,6)$ & $(28.2-41.5)$ \\
$80+$ & $56(27,0)$ & $(21.0-33.5)$ \\
Gênero & & \\
$\quad$ Masculino & $70(33,6)$ & $(26.8-40.0)$ \\
Feminino & $138(66,8)$ & $(60.0-73.2)$ \\
Procedência & & \\
Capital & $200(96,2)$ & $(92.6-98.6)$ \\
Interior & $8(3,8)$ & $(1.7-7.4)$ \\
\hline
\end{tabular}

*IC- intervalo de confiança nervoso central, os subgrupos terapêuticos mais frequentes foram os psicoanalépticos $(13,8 \%)$, os antiparkinsonianos $(5,2 \%)$ e os psicolépticos (5,3\%) (Tabela 2).

\section{Inadequações em prescrições medicamentosas}

O número de medicamentos prescritos variou de 2 a 10, num total de 794 especialidades farmacêuticas; verificou-se que as mulheres utilizaram em média 3,8 $\pm 1,8$ semelhante à média dos homens, que é de 3,9 $\pm 1,4$ medicamentos. A média por paciente foi de $3,8 \pm 1,6$ medicamentos. $\mathrm{Na}$ Tabela 3, observa-se que $45(21,6 \%)$ idosos utilizavam 2 medicamentos; 66 (31,7\%) utilizavam três; $34(16,4 \%)$ utilizavam quatro medicamentos, e $63(30,3 \%)$ idosos faziam o uso de 5 ou mais medicamentos, caracterizando a polifarmácia.

Dentre as 208 prescrições analisadas, foram encontradas duplicidade terapêutica em duas $(0,9 \%)$, uma referente à nortriptilina (N06) e paroxetina (N06) para um paciente de 62 anos, e outra de venlafaxina (N06) e amitriptilina (N06) para um paciente de 65 anos, sendo que todos esses medicamentos pertencem ao mesmo subgrupo terapêutico dos psicoanalépticos de acordo com a classificação ATC.

Em relação às potenciais interações medicamentosas, foram identificadas 406 ocorrências em 140 pacientes; uma média de $2,7 \pm 2,1$ por paciente idoso, sendo no mínimo uma e no máximo quinze ocorrências destas entre os idosos. A maior parte delas possuía gravidade moderada ( $83 \%)$, as de classificação da gravidade maior somaram 3\% das ocorrências, e as de gravidade menor, 14\% (Gráfico 1).

Das classes terapêuticas mais envolvidas em interações medicamentosas, destacaram-se: medicamentos cardiovasculares $(35,0 \%)$ e anti-inflamatórios não esteroidais $(23,4 \%)$. As IM mais frequentes foram entre captopril/Ácido Acetil Salicílico (3,7\%), captopril/hidroclorotiazida e captopril/Glibenclamida (2,7\%). Quanto à gravidade das IM classificadas em maior, foram detectadas entre Captopril/hidroclorotiazida (1,2\%), haloperidol/clorpromazina $(0,2 \%)$, nortriptilina/ paroxetina $(0,2 \%)$, prometazina/haloperidol $(0,5 \%)$, sertralina e selegilina $(0,2 \%)$, venlafaxina/ amitriptilina $(0,2 \%)$.

Analisando-se os medicamentos prescritos quanto à sua inadequação para pacientes idosos, segundo critérios de Beers, foram encontradas 52 ocorrências dentre os 794 medicamentos prescritos (6,5\%). Em 52 (25\%) pacientes foi observada 
Tabela 2. Distribuição dos principais medicamentos prescritos segundo a classificação ATC $^{*}$ dos idosos atendidos $(\mathrm{n}=208)$ no AG/HUJBB, 2011.

\begin{tabular}{|c|c|c|}
\hline & Categoria Terapêutica & n (\%) \\
\hline A & Aparelho digestório e metabólico & $111(14,0)$ \\
\hline $\mathrm{B}$ & Sangue e órgão hematopoiéticos & $93(11,7)$ \\
\hline $\mathrm{C}$ & Aparelho cardiovascular & $293(37,0)$ \\
\hline $\mathrm{C} 01$ & Terapêutica Cardíaca & $18(2.2)$ \\
\hline $\mathrm{C} 02$ & Anti-hipertensores & $3(0.4)$ \\
\hline $\mathrm{C} 03$ & Diuréticos & $58(7.3)$ \\
\hline $\mathrm{C} 04$ & Vasodilatadores periféricos & $1(0.1)$ \\
\hline $\mathrm{C} 07$ & Betabloqueadores & $22(2.8)$ \\
\hline $\mathrm{C} 08$ & Bloqueadores dos canais de cálcio & $38(4.7)$ \\
\hline $\mathrm{C} 09$ & Agentes que atuam sobre o sistema renina-angiotensina & $118(14.8)$ \\
\hline $\mathrm{C} 10$ & Hipolipemiantes & $34(4.3)$ \\
\hline $\mathrm{D}$ & Terapia dermatológica & $5(0,6)$ \\
\hline $\mathrm{H}$ & Terapia hormonal & $14(1,8)$ \\
\hline $\mathrm{J}$ & Terapia anti-infecciosa (Uso sistêmico) & $18(2,3)$ \\
\hline $\mathrm{L}$ & Terapia antineoplásica e agentes imunomoduladores & $9(1,1)$ \\
\hline $\mathrm{M}$ & Sistema musculoesquelético & $16(2,0)$ \\
\hline $\mathrm{N}$ & Sistema nervoso central & $219(27,5)$ \\
\hline N02 & Analgésicos & $9(1.0)$ \\
\hline N03 & Antiepiléticos & $13(1.6)$ \\
\hline N04 & Antiparkinsonianos & $41(5.2)$ \\
\hline N05 & Psicolépticos & $42(5.3)$ \\
\hline N06 & Psicoanalépticos & $110(13.8)$ \\
\hline N07 & Outros medicamentos do sistema nervoso & $4(0.5)$ \\
\hline $\mathrm{P}$ & Antiparasitários, inseticidas e repelentes & $8(1,0)$ \\
\hline $\mathrm{R}$ & Aparelho respiratório & $10(1,2)$ \\
\hline$S$ & Órgãos dos sentidos & $2(0,2)$ \\
\hline Total & & $794(100,0)$ \\
\hline
\end{tabular}

*ATC: Classificação Anatômica-Terapêutica-Química

Tabela 3. Distribuição percentual dos medicamentos prescritos para os pacientes idosos atendidos $(\mathrm{n}=$ 208) no AG/HUJBB, 2011.

\begin{tabular}{crr}
\hline Número de medicamentos prescritos & n & $\%$ \\
\hline 2 & 45 & 21,6 \\
3 & 66 & 31,7 \\
4 & 34 & 16,4 \\
5 & 31 & 14,9 \\
6 & 19 & 9,1 \\
7 & 7 & 3,4 \\
8 & 3 & 1,4 \\
9 & 2 & 1,0 \\
10 & 1 & 0,5 \\
Total & 208 & 100,0
\end{tabular}

prescrição de pelo menos um medicamento inapropriado, sendo que 33 (15,9\%) ocorrências foram encontradas entre as mulheres e $19(9,1 \%)$ entre os homens. $\mathrm{O}$ medicamento inapropriado mais prescrito foi o nifedipino (2,8\%) (Tabela 4).

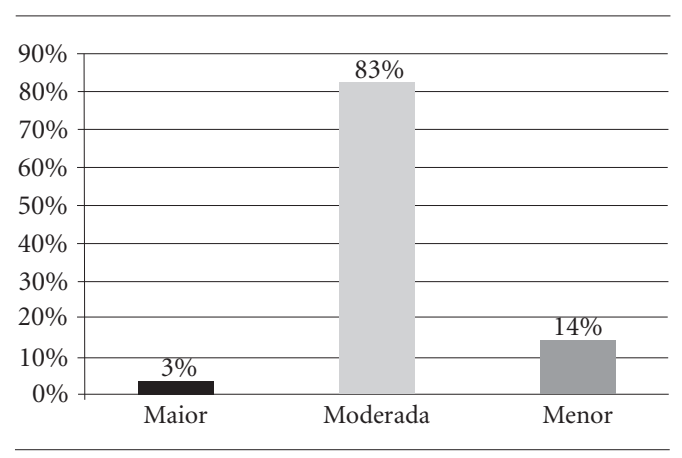

Gráfico 1. Estratificação das potenciais interações medicamentosas $(n=406)$ de acordo com a gravidade.

\section{Discussão}

As mulheres representam 66,8\% dos entrevistados, tal predominância tem sido recorrente em outros estudos científicos ${ }^{2,11,28}$ que também detectaram que as idosas possuidoras de comorbidades au- 
Tabela 4. Medicamentos classificados como potencialmente inapropriados prescritos para idosos, segundo o critério de Beers $201217\left(\mathrm{n}=51^{\star}\right)$ no AG/HUJBB, 2011.

\begin{tabular}{|c|c|c|c|c|}
\hline Medicamento & n (\%) & Possíveis consequências & Recomendação & $\begin{array}{l}\text { Qualidade } \\
\text { da evidência }\end{array}$ \\
\hline Amiodarona & $3(0,4)$ & $\begin{array}{l}\text { Alterações do intervalo QT; arritmias } \\
\text { graves como torsades de pointes. }\end{array}$ & $\begin{array}{l}\text { Evitar drogas } \\
\text { antiarrítmicas como } \\
\text { primeira linha para } \\
\text { tratamento de fibrilação } \\
\text { atrial. }\end{array}$ & Alta \\
\hline Amitriptilina & $7(0,9)$ & $\begin{array}{l}\text { Propriedades anticolinérgicas e } \\
\text { sedativas. }\end{array}$ & Evitar & Alta \\
\hline Diazepam & $8(1,0)$ & $\begin{array}{l}\text { Meia-vida longa, sedação prolongada, } \\
\text { aumentam o risco de quedas e fraturas. }\end{array}$ & $\begin{array}{l}\text { Evitar benzodiazepínicos } \\
\text { para o tratamento de } \\
\text { insônia, agitação ou delírio. }\end{array}$ & Alta \\
\hline Digoxina & $9(1,1)$ & $\begin{array}{l}\text { Redução da depuração renal leva a } \\
\text { maior risco de efeitos tóxicos. }\end{array}$ & Evitar & Moderada \\
\hline Metildopa & $3(0,4)$ & $\begin{array}{l}\text { Pode causar bradicardia e exacerbar } \\
\text { depressão em pacientes mais velhos. }\end{array}$ & Evitar & Baixa \\
\hline $\begin{array}{l}\text { Nifedipino } \\
\text { curta ação }\end{array}$ & $19(2,4)$ & $\begin{array}{l}\text { Potencial para hipotensão e } \\
\text { constipação. }\end{array}$ & Evitar & Alta \\
\hline Prometazina & $3(0,4)$ & Efeitos anticolinérgicos & Evitar & Alta \\
\hline Total & $52(6,5)$ & & & \\
\hline
\end{tabular}

mentam o risco para utilização de cuidados de saúde. Isso ocorre possivelmente porque as mulheres apresentam pior estado funcional e saúde autorreferida, sintomas depressivos e hospitalizações ${ }^{29}$, aparecendo como maiores consumidoras de medicamentos prescritos ${ }^{30}$. Em uma revisão sobre fatores associados ao mau uso de medicamentos em idosos realizado por Rozenfeld ${ }^{22}$, apontou que o consumo de medicamentos é maior entre as mulheres idosas sendo influenciados por fatores preditores, como idade avançada, piores condições de saúde e depressão.

No entanto, observou-se neste estudo que a média de medicamentos prescritos entre homens e mulheres foi semelhante provavelmente porque a amostra deste estudo é pequena e restrita aos idosos atendidos no ambulatório de geriatria do HUJBB.

A polifarmácia foi identificada em 30,3\% prescrições geriátricas, sendo a média de 3,8 medicamentos/idoso; dado semelhante foi encontrado nos estudos de Flores e Mengue ${ }^{19}$, com idosos do município da região Sul do Brasil, onde 27,0\% dos idosos foram caracterizados com polifarmácia e o número médio de medicamentos consumidos foi igual a 3,2 e na pesquisa de Galato et al. ${ }^{31} \mathrm{com}$ residentes em uma cidade do sul de Santa Catarina, que utiliza em média 3,5 medicamentos/idoso. Pode-se dizer, entre outros fatores, que a elevada prevalência da polifarmácia entre os idosos está associada ao número de diagnósticos médicos. Assim, quanto maior o número de problemas médicos identificados, maior a lista de prescrições ${ }^{30}$.

Os medicamentos utilizados para problemas do sistema cardiovascular foram os mais prescritos $(37,0 \%)$, esta característica da utilização de medicamentos dos participantes foi semelhante ao observado em outros estudos nacionais ${ }^{32-34}$. Isso reflete a alta prevalência de doenças cardiovasculares, que vêm liderando as causas de morbimortalidade, entre a população idosa, com atenção especial para hipertensão arterial sistêmica (HAS), que tem atingindo $50 \%$ dos indivíduos com mais de 65 anos, além das alterações cardíacas próprias do envelhecimento ${ }^{33}$.

Outro grupo de medicamentos que apresentou um número alto de prescrições para essa faixa etária foram os que atuam no sistema nervoso central (27,5\%), semelhante aos estudos de Coelho Filho et al..$^{30}$, no qual o uso de medicamentos para o sistema nervoso foi de $13,5 \%$ e no de Aguiar et al. ${ }^{35}$ com 20,1\%. Tais medicamentos são utilizados com frequência pelos idosos estudados, em virtude de estes serem acometidos por doenças do tipo neurodegenerativas e depressão.

Entre os 208 pacientes estudados, identificaram-se 406 interações medicamentosas, sendo aproximadamente duas IM por paciente. Locatelli ${ }^{36}$ obteve uma média de quatro potenciais IM por paciente estudado A classe mais envolvida 
nas interações neste estudo foi de fármacos para terapia cardíaca $(35,0 \%)$, o que também foi verificado por Simões e Marques ${ }^{37}$. A interação medicamentosa mais frequente neste estudo foi entre o captopril e o ácido acetilsalicílico, que é de gravidade moderada; o principal efeito desta interação é a redução do efeito anti-hipertensivo do inibidor da enzima conversora de angiotensina devido à inibição da síntese de prostaglandinas pelo ácido acetilsalicílico ${ }^{26}$.

A existência de interações medicamentosas representa um problema que deve ser tratado com cautela por médicos e farmacêuticos. Muitas dessas interações devem ser absolutamente evitadas, principalmente em pacientes idosos, que, de forma geral, são mais sensíveis aos efeitos terapêuticos e tóxicos dos medicamentos ${ }^{38}$.

Os medicamentos comumente envolvidos nas potenciais interações medicamentosas relevantes são aqueles usados no cotidiano do manejo do paciente idoso com doenças crônicas ${ }^{36}$, sendo que no presente estudo a maior parte destas IM foram classificadas com moderadas que exige monitorização constante, pois os efeitos destas interações podem causar uma piora do estado clínico do paciente que pode resultar na necessidade de tratamento adicional, hospitalização, custos ao sistema de saúde, ou se o paciente já estiver internado, levar a um tempo maior de internação. Uma solução para esse problema pode ser a seleção de um fármaco que não produza interação (ex: substituição de fluoxetina por outro Inibidor Seletivo da Recaptação de Serotonina), contudo, se não houver esta alternativa, é importante administrar os medicamentos que interagem entre si sob o monitoramento realizado através da prática da farmácia clínica.

Utilizando o critério de Beers atualizado ${ }^{17}$, foi encontrada a proporção de $6,5 \%$ de pacientes que apresentou na sua prescrição pelo menos um medicamento inadequado. O nifedipino, que é um antagonista do canal de cálcio (ACC), foi o medicamento inadequado mais frequente, sendo usado por aproximadamente $2,4 \%$ dos idosos entrevistados; dados semelhantes também foram encontrados no estudo realizado por Correr et al. ${ }^{38}$, o qual apontou como medicamento inadequado mais utilizado o nifedipino (25,8\%). Esse medicamento é considerado inadequado para o idoso, devido ao seu alto potencial de risco para o desenvolvimento de hipotensão e constipação intestinal em idosos ${ }^{16}$. Assim sendo o efeito adverso de um medicamento inadequado, é interpretado incorretamente como nova condição médica que exige nova prescrição, sendo o paciente exposto ao risco de desenvolver efeitos prejudiciais adicionais relacionados ao tratamento potencialmente desnecessário, efeito que é conhecido como "cascata da prescrição" 39 .

O uso de medicamentos inadequados continua sendo frequente em prescrições para idosos, como pode ser encontrado em estudos internacionais ${ }^{27,40-42}$. A ausência de alternativas terapêuticas mais custo-efetivas e com melhor perfil de segurança para esses pacientes nos serviços públicos de saúde podem favorecer a prescrição de medicamentos inapropriados ${ }^{13}$.

Deve-se isso aos modelos de estudos de medicamentos que excluem pacientes idosos de ensaios clínicos. Naturalmente, os resultados desses ensaios em pessoas jovens não podem ser extrapolados para pessoas idosas. Quando os “idosos" participam da fase III, estão com 60 anos no máximo e são saudáveis, sendo, porém, inadequadamente representados.

Os dados desse estudo demonstram a existência de inadequações potenciais na prescrição medicamentosa de pacientes idosos atendidos no ambulatório de geriatria de um hospital universitário. Os resultados podem ser úteis para o desenvolvimento de mecanismos de monitoramento e avaliação do processo da prescrição que visem a reduzir essas inadequações, aumentando a oportunidade de se obter resultados terapêuticos positivos e benéficos para esses pacientes.

O uso irracional de medicamentos em idosos é um importante problema de saúde pública na medida que expõe os mesmos aos riscos potenciais dos medicamentos, o envolvimento do farmacêutico clínico na equipe multiprofissional de geriatria pode qualificar o atendimento personalizado, através da implantação de protocolos e procedimentos que visem minimizar e evitar as inadequações potenciais em prescrições. A prescrição de qualidade representa um dos componentes do uso racional, contribuindo para a adesão e efetividade do tratamento, garantindo a segurança do paciente idoso.

Neste contexto nosso estudo apresenta evidência científica considerando as pesquisas já realizadas sobre o tema, entretanto a contribuição deste estudo em nível ambulatorial pode subsidiar ações da assistência farmacêutica na construção de uma linha de cuidados a pessoa idosa.

\section{Conclusão}

Este trabalho caracterizou esta amostra como a maioria dos entrevistados sendo mulheres, com faixa etária 60 a 69 anos, a média de idade dos 
pacientes foi de 73,4 anos, e grande parte procedente da capital.

Entre os medicamentos mais utilizados destacam-se os prescritos para o sistema cardiovascular, especialmente os que atuam sobre o sistema renina angiotensina; a prática da polifarmácia foi observada em 30,3\% dos entrevistados. Foi detectada duplicidade terapêutica relacionada a medicamentos que atuam no sistema nervoso central, na prescrição dos pacientes idosos. Potencias interações medicamentosas foram encontradas em 67,3\% prescrições, bem como o uso de medicamentos inapropriados para $25 \%$ dos idosos.

O estudo sobre a temática de uso de medicamentos por idosos, a identificação de polifarmácia, interações medicamentosas, prescrição de medica- mentos inapropriados e duplicidade terapêutica, representam fatores que influenciam diretamente na qualidade de vida do idoso, seja de forma isolada ou associadas a alterações fisiológicas e as comorbidades, reforçando a importância da avaliação do plano terapêutico, considerando a sua complexidade, influenciando assim na tomada de decisão clínica, que identifique riscos ou danos em potencial relacionados aos medicamentos prescritos.

Assim é imprescindível que se assuma a promoção da racionalidade terapêutica como elemento de preocupação e atenção da equipe multiprofissional de saúde para garantir a segurança do paciente idoso com uma farmacoterapia racional e segura, que venha expressar um indicador de qualidade na assistência ao idoso.

\section{Colaboradores}

VS Cuentro, LF Gerlack participaram da redação do artigo, AJG Bós e AF Oliveira participaram na Análise e interpretação dos dados, MAA Silva participou na revisão crítica relevante do conteúdo intelectual e MA Andrade participou na aprovação final da versão a ser publicada.

\section{Agradecimentos}

Ao Programa de Residência Multiprofissional em Saúde do Idoso do Ministério da Educação (MEC), vinculado ao HUJBB/UFPA, pela concessão de bolsa de estudo. 


\section{Referências}

1. Fundação Instituto Brasileiro de Geografia e Estatística (IBGE). Síntese dos Indicadores Sociais: uma análise das condições de vida da população brasileira 2010 [Internet]. 2010 [acessado $2012 \mathrm{fev} 28$ ]. Disponível em: http://www.ibge.gov.br/home/estatistica/populacao/ condicaode vida/indicadoresminimos/sinteseindicsociais2010/SIS_2010.pdf

2. Marin MJS, Cecílio LCO, Perez AEWUF, Santella F, Silva CBA, Filho JRG, Roceti LC. Caracterização do uso de medicamentos entre idosos de uma unidade do Programa Saúde da Família. Cad Saude Publica 2008; 24(7):1545-1555.

3. Hajjar ER, Cafiero AC, Hanlon JT. Polypharmacy in the elderly patients. Am J Geriatr Pharmacother 2007; 5(4):345-351.

4. Nguyen JK, Fours MM, Kotabe SE, Lo E. Polypharmacy as a risck factor for adverse drug reactions in geriatric nursing home residents. Am J Geriatr Pahrmacother 2006; 4(1):36-41.

5. Galvão MPA, Ferreira MBC. Prescrição de medicamentos em Odontogeriatria. In: Wannmacher L, Ferreira MBC editores. Farmacologia Clínica para dentistas. Rio de Janeiro: Guanabara Koogan; 2007. p. 440-452.

6. Secoli SR. Polifarmácia: interações e reações adversas no uso de medicamentos por idosos. Rev. bras. Enferm 2010; 63(1):136-140.

7. Hammes JA, Pfuetzenreiter F, Da Silveira F, Koenig A, Westphal GA. Prevalência de potenciais interações medicamentosas droga-droga em unidades de terapia intensiva. Rev Bras Ter Intensiva 2008; 20(4):349-354.

8. Bagatini F, Blatt CR, Maliska G, Trespash GV, Pereira IA, Zimmermann AF, Storb BH, Farias MR. Potenciais Interações medicamentosas em pacientes com artrite reumatoide. Rev Bras Reumatol 2011; 51(1):20-39.

9. Katzung BG. Aspectos especiais da farmacologia geriátrica. In: Katzung BG, organizador. Farmacologia básica \& clínica. Rio de Janeiro: Editora Guanabara-Koogan; 2002. p. 889-906.

10. Nobrega OT, Karnikowski, MGO. A terapia medicamentosa no idoso: cuidados na medicação. Cien Saude Colet 2005; 10(2):309-313.

11. Golden AG, Qiu D, Roos BA. Medication assessments by care managers reveal potential safety issues in homebound older adults. Ann Pharmacother 2011; 45(4):492-498.

12. O'Mahony D, Gallagher PF. Inappropriate prescribing in the older population: need for new criteria. Age Ageing 2008; 37(2):138-141.

13. Oliveira MG, Amorim WW, Rodrigues VA, Passos LC. Access of Brazilian elders to potentially inappropriate medications. Rev APS 2011; 14(3):258-265.

14. Beers M, Ouslander J, Rollingher I, Reuben D, Brooks J, Beck J. Explicit criteria for determining inappropriate medication use in nursing home residents. Ucla division of geriatric medicine. Arch Intern Med 1991; 151(9):1825-1832.

15. Beers MH. Explicit criteria for determining potentially inappropriate medication use by the elderly. An update. Arch Intern Med 1997; 157(14):1531-1536.
16. Fick DM, Cooper JW, Wade WE, Waller JL, Maclean JR, Beers MH. Updating the Beers criteria for potentially inappropriate medication use in older adults: results of a US consensus panel of experts. Arch Intern Med 2003; 163(22):2716-2724.

17. The American Geriatrics Society 2012 Beers Criteria Update Expert Panel. AGS updated Beers Criteria for potentially inappropriate medication use in older adults. J Am Geriatr Soc 2012; 60(4):616-631.

18. Acurcio FA, Silva AL, Ribeiro AQ, Rocha NP, Silveira MR, Klein CH, Rozenfeld S. Complexidade do regime terapêutico prescrito para idosos, Belo Horizonte, MG. Rev Assoc Med Bras 2009; 55(4):468-474.

19. Flores LM, Mengue SS. Uso de medicamentos por idosos em região do sul do Brasil. Rev Saude Publica 2005; 39(6):924-929.

20. Pereira LRL, Vecchi LUP, Baptista MEC, Carvalho D. Avaliação da utilização de medicamentos em pacientes idosos por meio de conceitos de farmacoepidemiologia e farmacovigilância. Cien Saude Colet 2004; 9(2):479-481.

21. Ribeiro AQ, Acurcio FA, Wick JY. Pharmacoepidemiology of the elderly in Brazil: state of the art. Consult Pharm 2009; 24(1):30-44.

22. Rozenfeld S. Prevalência, fatores associados a mau uso de medicamentos entre idosos: uma revisão. Cad Saude Publica 2003; 19(3):717-724.

23. Silva CSO, Pereira MI, Yoshitome AY, Rodrigues Neto JF, Barbosa DA. Avaliação do uso de medicamentos pela população idosa em Montes Claros, Minas Gerais, Brasil. Esc Anna Nery 2010; 14(4):811-818.

24. World Health Organization (WHO). ATC Index with DDD 2008 [online]. Oslo: WHO; 2008. [acessado 2012 fev 10]. Disponível em: http://www.whocc.no/ atc_ddd_index/.

25. Secoli SR, Duarte YAO. Medicamentos e a assistência domiciliária. In: Duarte YAO, Diogo MJD. Atendimento domiciliar: um enfoque gerontológico. São Paulo: Atheneu; 2000. p. 326-335.

26. Micromedex ${ }^{\circledR}$ Healthcare Series [database on the Internet]. Versão 5.1. Greenwood Village, Colo: Thomson Micromedex; 2011.

27. Brasil. Ministério da Saúde (MS). Conselho Nacional de Saúde. Resolução nº 196 de 10 de outubro de 1996. Diretrizes e Normas Regulamentadoras de Pesquisas Envolvendo Seres Humanos. Diário Oficial da União 1996; 16 out.

28. Fick D, Mion L, Beers M, Waller JL. Health outcomes associated with potentially inappropriate medication use in older adults. Res Nurs Health 2008; 31(1):42-51.

29. Chrischilles EA, Foley DJ, Wallace RB, Lemcke JH, Semla TP, Hanlon JT, Glynn RJ, Ostfeld AM, Guralnik JM. Use of medications by persons 65 and over: Data from the established populations for epidemiologic studies of the elderly. J Gerontol 1992; 47(5):137-144.

30. Coelho Filho JM, Marcopito LF, Castelo A. Perfil de utilização de medicamentos por idosos em área urbana do nordeste do Brasil. Rev Saude Publica 2004; 38(4):557-564. 
31. Galato D, Silva ES, Tiburcio LS. Estudo de utilização de medicamentos em idosos residentes em uma cidade do sul de Santa Catarina (Brasil): um olhar sobre a polimedicação. Cien Saude Colet 2010; 15(6):2899-2905.

32. Ribeiro AQ, Rozenfeld S, Klein CH, César CC, Acurcio FA. Inquérito sobre uso de medicamentos por idosos aposentados, Belo Horizonte, MG. Rev Saude Publica 2008; 42(4):724-732.

33. Rozenfeld S, Fonseca MJM, Acurcio FA. Drug utilization and polypharmacy among the elderly: a survey in Rio de Janeiro City, Brazil. Rev Panam Salud Pública 2008; 23(1):34-43.

34. Vosgerau M, Soares D, Souza R, Matsuo T, Carvalho G. Consumo de medicamentos entre adultos na área de abrangência de uma Unidade de Saúde da Família. Cien Saude Colet 2011; 16(1):1629-1638.

35. Aguiar PM, Lyra Junior DP, Silva DT, Marques TC. Avaliação de farmacoterapia de idosos residentes em instituição asilares no nordeste do Brasil. Lat Am J Pharm 2008; $27(3): 454-459$.

36. Locatelli J. Interações medicamentosas em idosos hospitalizados. Hospital Israelita Albert Einstein 2007; 5(4):343-346.

37. Simões MJS, Marques AC. Consumo de medicamentos por idosos segundo prescrição médica em Jaú-SP. Rev Ciênc Farm Básica Apl 2005; 26(2):139-144.

38. Correr CJ, Pontarolo R, Ferreira LC, Baptistão SAM. Riscos de problemas relacionados com medicamentos em pacientes de uma instituição geriátrica. Rev Bras Cienc Farm 2007; 43(1):55-62.

39. Rochon PA, Gurwitz JH. Optimising drug treatment for elderly people:the prescribing cascade. BMJ 1997; 315(7115):1096-1099.

40. De Wilde S, Carey I, Harris T, Richards N, Victor C, Hilton S, Cook D. Trends in potentially inappropriate prescribing amongst older UK primary care patients. Pharmacoepidemiol Drug Saf 2007; 16(6):658-667.

41. Ryan C, O’Mahony D, Kennedy J, Weedle P, Byrne S. Potentially inappropriate prescribing in an Irish elderly population in primary care. Br J Clin Pharmacol 2009; 68(6):936-947.

42. Dimitrow MS, Airaksinen MS, Kivelä SL, Lyles A, Leikola $\mathrm{SN}$. Comparison of prescribing criteria to evaluate the appropriateness of drug treatment in individuals aged 65 and older: a systematic review. J Am Geriatr Soc 2011; 59(8):1521-1530.

Artigo apresentado em 21/06/2013

Aprovado em 15/08/2013

Versão final apresentada em 21/08/2013 